\title{
Musashi-2 promotes migration and invasion in bladder cancer via activation of the JAK2/STAT3 pathway
}

\author{
Chenlu Yang ${ }^{1,2,4}$, Weijing Zhang ${ }^{2,4}$, Longwang Wang ${ }^{1,3,4}$, Gallina Kazobinka ${ }^{1}$, Xiaomin Han ${ }^{1}$, Bin Li and Teng Hou ${ }^{1,2}$
}

Musashi-2 (Msi2) is considered to have a crucial role in regulating various key cellular functions. However, the clinical significance and biological role of Msi2 in bladder cancer remains unknown. We examined the expression of Msi2 in bladder cancer cell lines in 167 clinical samples and the biological role of Msi2 in bladder cancer cells. Western blotting was used to investigate the possible mechanism of Msi2-induced migration and invasion in bladder cancer. Msi2 was significantly upregulated in bladder cancer cells and tissues compared with normal bladder urothelial cells and tissues. Immunohistochemical analysis revealed high expression of Msi2 in 57 of 167 (34.1\%) bladder cancer specimens. Statistical analysis showed a significant correlation of Msi2 expression with advanced clinical stage, lymph node metastasis, and poor prognosis. Overexpression and ablation of Msi2 promoted and inhibited, respectively, the migration and invasion of bladder cancer cells. Furthermore, we found that Msi2 activated the JAK2/STAT3 pathway and promoted expression of genes downstream of JAK2/STAT3 in bladder cancer. This study demonstrates that Msi2 can induce bladder cancer cell migration and invasion by activating the JAK2/STAT3 pathway, and that Msi2 may be a valuable prognostic biomarker for bladder cancer patients.

Laboratory Investigation (2016) 96, 950-958; doi:10.1038/labinvest.2016.71; published online 20 June 2016

Bladder cancer is the most common malignancy of the urinary tract and the ninth most common cancer worldwide, with $\sim 430000$ new cases and 165000 deaths annually. ${ }^{1}$ Despite the recent advances in treatments, the prognosis of patients with advanced-stage bladder cancer remains poor. ${ }^{2}$ The 5-year overall survival rate for early stage bladder cancer is more than $80 \%$, compared with only $20 \%$ for advanced stage disease. ${ }^{3}$ In patients with lymph node-positive disease, the 5 -year overall survival rate is $26 \% .{ }^{4}$ Although metastatic bladder cancer remains a deadly disease, there are neither efficient therapeutic options for curative treatment, nor sensitive molecular biomarkers for predicting tumor metastasis before they become evident.

Musashi-2 (Msi2) is a member of the Musashi family that contains two RNA recognition motifs and can be expressed as more than one isoform due to alternative splicing. ${ }^{5}$ The Msi2 gene is located at chromosome $17 \mathrm{q} 22$ and has a sequence length of $987 \mathrm{bp} .{ }^{6}$ As a RNA-binding protein, Msi2 is linked to stem cells and contribute to asymmetric cell division control, germ and somatic stem cell function, and cell fate determination in a number of tissues. ${ }^{7}$ Msi2 has been shown to contribute to specific neuronal lineages generation and maintenance. ${ }^{8}$ In addition, Msi2 has been demonstrated as an important modulator of hematopoietic stem cells (HSCs) and their progenitors. Knockdown of Msi2 leads to reduced engraftment and depletion of HSCs in vivo. ${ }^{9}$ More recent studies showed that Msi2 may have a unique role that is required for normal spermatogenesis, with Msi2 overexpression resulting in male sterility in vivo. ${ }^{10}$

Apart from being a cell development regulator, Msi2 is also linked to carcinogenesis and tumor progression. Recent studies have shown that Msi2 is a mediator of proliferation and differentiation in myeloid malignancies, ${ }^{9}$ and its knockdown leads to cell cycle arrest and cell apoptosis in acute myeloid leukemia (AML). ${ }^{11}$ Besides, Msi2 could be rearranged to form a fusion gene with HOXA9, which might have an important role in disease progression of chronic myeloid leukemia. ${ }^{12}$ Similarly, Msi2 has been proposed to correlate

\footnotetext{
${ }^{1}$ Department of Urology, Union Hospital, Tongji Medical College, Huazhong University of Science and Technology, Wuhan, Hubei, China; ${ }^{2}$ State Key Laboratory of Oncology in South China, Sun Yat-sen University Cancer Center, Collaborative Innovation Center for Cancer Medicine, Guangzhou, Guangdong, China and ${ }^{3}$ Department of Urology, The Second Affiliated Hospital of Nanchang University, Nanchang, Jiangxi, China

Correspondence: Dr T Hou, MD, PhD, Department of Urology, Union Hospital, Tongji Medical College, Huazhong University of Science and Technology, Wuhan, Hubei 430022, China.

E-mail: 1aiyan@163.com

${ }^{4}$ These authors contributed equally to this work.
}

Received 5 February 2016; revised 23 May 2016; accepted 23 May 2016 
with epithelial-mesenchymal transition in hepatocellular carcinoma (HCC). ${ }^{13}$

In light of these findings, we investigated the expression profile of Msi2 in bladder cancer and report here, for the first time, that Msi2 expression is closely correlated with clinicopathologic features in bladder cancer patients, and that Msi2 might promote cell migration and invasiveness by activating the JAK2/STAT3 pathway. Thus, Msi2 may have a crucial role in bladder cancer progression and it could be a promising prognostic marker and a therapeutic target.

\section{MATERIALS AND METHODS \\ Cell Lines}

Primary cultures of normal bladder urothelial cells (NBUCs) were established from fresh specimens of patients as described previously. ${ }^{14}$ Human bladder cancer cell lines, including BIU-87, J82, 5637, EJ, and RT4 were obtained from the Cell Bank of the Chinese Academy of Sciences (Shanghai, China) and cultured in RPMI 1640 medium supplemented with 10\% fetal bovine serum (FBS; Gibco, Grand Island, NY, USA).The UM-UC-3 cell line was cultured in Dulbecco's Modified Eagle's Medium was obtained from the Cell Bank of the Chinese Academy of Sciences and cultured in McCoy's 5a Medium supplemented with 10\% FBS (Gibco).

\section{Vectors and Lentiviral Infection}

A Msi2 expression construct was generated by subcloning PCR-amplified full-length human Msi2 cDNA into the pReceiver-Lv121 plasmid (ordered from Genecopoeia, cat. no. EX-T2560-Lv121). The length of Msi2 open reading frame (ORF) was $987 \mathrm{bp}$. To deplete Msi2, two human Msi2-targeting siRNA sequences were synthesized and purified by Ribobio (Guangzhou, Guangdong, China), The siRNA sequences used were as follows: RNAi\#1: 5'-CAAUGCUGAUGUUUGAUAA dTdT-3' and RNAi\#2: 5'-GGAGAAAGUCUGUGAGAUUdT dT-3'. Stable cell lines expressing Msi2 and Msi2-shRNA were generated by lentiviral infection. For lentiviral production, the lentiviral expression vector was co-transfected with lentivirus packing vectors into 293T cells using Lipofectamine Reagent (Invitrogen, Carlsbad, CA, USA). Stable cell lines expressing Msi2 were selected for 3 days with $1.0 \mathrm{mg} / \mathrm{ml}$ puromycin.

\section{Real-Time PCR}

RNAs were extracted from cells and fresh tissues using Trizol (Invitrogen) kit according to the manufacturer's instructions. Total RNA was then reversely transcribed using RevertAid First Strand cDNA SynthesisKit (Thermo Scientific, MA, USA). Subsequently, real-time PCR was then performed in Bio-Rad system (Bio-Rad System, CA, USA). The expression level of Msi2 gene was normalized by GAPDH. The PCR primers sequences were: Msi2, sense, 5'-AGCACGACCCCGG TAAAATG-3', and anti-sense, 5'-GCTCGACGAGGAAATGC AAC-3'; GAPDH, sense, 5' - CGAGATCCCTCCAAAATCAA -3', and anti-sense, 5'- TTCACACCCATGACGAACAT - $3^{\prime}$. The
$2-\Delta \Delta \mathrm{CT}$ method was used to calculate relative expression of Msi2 mRNA.

\section{Western Blotting}

Cells and tissue samples were lysed in RIPA lysis buffer, and the protein concentrations were detected using a BCA Protein Assay Kit (Thermo Scientific). Cell/tissue lysates were separated by SDS-PAGE gels and transferred to a polyvinylidene difluoride (PVDF) membrane (Millipore, Eschborn, Germany). The blots were blocked in $5 \%$ milk for $1 \mathrm{~h}$ at room temperature. PVDF membranes were incubated with the primary antibodies overnight in a cold room at $4{ }^{\circ} \mathrm{C}$. Subsequently, bound primary antibodies were reacted with corresponding secondary antibodies for $2 \mathrm{~h}$ at room temperature and detected by chemiluminescence. The anti-Msi2 (cat. no. ab76148), anti-JAK2 (cat. no. ab108596), anti-phospho-JAK2 (cat. no. ab32101), anti-STAT3 (cat. no. ab68153), antiphospho-STAT3 (cat. no. ab76315), anti-WASF3 (cat. no. ab68031), and anti-GAPDH (cat. no. ab9484) antibodies were purchased from Abcam (Cambridge, UK).

\section{Cell Migration and Invasion Assays}

Migration of cells was assessed by using 24-well chambers with $8-\mu \mathrm{m}$ pore membranes (Corning LifeSciences, cat. no. 353097 ) and media containing $10 \%$ FBS in the lower chamber as a chemoattractant. Cells $\left(1 \times 10^{5}\right)$ were seeded serum-free medium into the upper chamber and allowed to invade toward $10 \%$ FBS in the lower chamber. After $24 \mathrm{~h}$, migratory cells were fixed and stained with $0.1 \%$ crystal violet. Migrating cells were measured by using image analysis software ImagePro Plus 6.0 (Media Cybernetics, Bethesda, USA). Each sample was assayed in triplicate. Cell invasion ability was measured in Matrigel-coated Transwell insert chambers (Corning LifeSciences, cat. no. 353097). The inserts were coated with $1 \mathrm{mg} / \mathrm{ml}$ Matrigel matrix according to the manufacturer's recommendations. Methods used in cell invasion assay were similar to those in the cell migration assay.

\section{Monolayer Scratch Wound Healing Assay}

Cells $\left(1 \times 10^{4}\right.$ per well $)$ were seeded in 12 -well plates and grown to confluence. After a scratch (wound) was created on each confluent monolayer using a $200-\mu \mathrm{l}$ sterile pipette tip, the cells were incubated $24 \mathrm{~h}$ in serum-free medium at a temperature-and $\mathrm{CO}_{2}$-controlled incubator (at $37^{\circ} \mathrm{C}, 5 \%$ $\mathrm{CO}_{2}$ ). Wound closure was investigated by measuring the distance between the opposite edges of the wound. At least three independent experiments were carried out.

\section{Colony Formation Assays}

Cells were plated on $60 \mathrm{~mm}$ dishes $\left(0.5 \times 10^{3}\right.$ cells per plate) and cultured at $37^{\circ} \mathrm{C}$ in $5 \% \mathrm{CO}_{2}$. After 10 days, colonies were stained with $1.0 \%$ crystal violet for $30 \mathrm{~s}$ after fixation with $10 \%$ formaldehyde for $5 \mathrm{~min}$. 


\section{MTT Assay}

Cells were seeded into 96-well plates at indicated time points with $100 \mathrm{ml}$ sterile MTT dye $(0.5 \mathrm{mg} / \mathrm{ml}$, Sigma $)$ for $4 \mathrm{~h}$ at $37^{\circ} \mathrm{C}$, the media was then removed and $150 \mu \mathrm{l}$ dimethyl sulfoxide (DMSO, Sigma) was added. The absorbance was measured at $570 \mathrm{~nm}$, with $655 \mathrm{~nm}$ as the reference wavelength. All experiments were performed in triplicate.

\section{Patient Information and Tissue Specimens}

This study was conducted on a total of 167 paraffinembedded, bladder cancer samples, which were histopathologically and clinically diagnosed at the Sun Yat-sen University Cancer Center between 2006 and 2009. The tumor stage was determined according to the TNM staging system of American Joint Committee on Cancer (AJCC). Prior written consent was obtained from all patients and this study was approved by the Research Ethics Committee of Sun Yat-sen University Cancer Center. Seven freshly collected bladder cancer tissues and matched adjacent non-tumor bladder tissues from the same patient were frozen and stored in liquid nitrogen until required. To select tumor adjacent normal tissue, grossly normal mucosa from the farthest resection margin was carefully excised and subjected to frozen section and evalution to exclude dysplasia and presence of inflammatory cells. The surface squamous epithelium of an adjacent area was then carefully peeled off from the submucosa and placed immediately in liquid nitrogen.

\section{Immunohistochemistry}

Immunohistochemistry (IHC) analysis was performed as previously described, ${ }^{15}$ using anti-Msi2 antibody (Abcam, Cambridge, cat. no. ab76148). The degree of immunostaining was evaluated by two independent observers who were blind to the clinical data of the patients. The scores were determined by combining the proportion of positively stained tumor cells and the intensity of staining. Intensity of stained cells was graded semi-quantitatively into four levels: 0 (no staining); 1 (weak staining =light yellow); 2 (moderate staining $=$ yellow brown) and 3 (strong staining $=$ brown); and the proportion was scored as: 0 , negative; $1,10 \%$ or less; $2,11-50 \%$; $3,51-80 \%$; or $4,80 \%$ or more positive cells. Intensity and fraction of positive cell scores were multiplied for each marker and thus the scoring system was defined as low expression for scores of $0-3$, and as high expression for scores of 4-12.

\section{Statistical Analysis}

All statistical analyses were conducted by using the SPSS 16.0 (Chicago, USA). Comparisons between groups for statistical significance were carried out with Student's $t$-test. The correlation between Msi2 expression and clinicopathologic characteristics was analyzed by the $\chi^{2}$ test. The overall survival and recurrence-free survival were calculated as the time from the date of primary surgery to the date of first death or recurrence. Survival curves were plotted by the Kaplan-Meier method and compared by using the log-rank test. Survival data were evaluated by using multivariate Cox regression analyses. $P<0.05$ was considered statistically significant in all cases.

\section{RESULTS}

\section{Msi2 is Upregulated in Bladder Cancer}

Expression of Msi2 was examined in bladder cancer cells and tissues. The expression of both the Msi2 protein and mRNA were markedly upregulated in bladder cancer cell lines, including BIU-87, J82, 5637, UM-UC-3, EJ, and RT4, in comparison with that in NBUCs (Figure 1a). Similarly, western blotting and real-time PCR showed that Msi2 expression was upregulated in bladder cancer tissue samples compared with that in paired adjacent non-tumor tissues (Figure $1 \mathrm{~b}$ ), indicating that Msi2 is upregulated in bladder cancer. In addition, western blotting showed that both bladder cancer cells and tissue express two isoforms (iso 1 and iso 2) of Msi2, and that isoform 2 appears to be the major isoform (Figure 1).

\section{Msi2 Expression is Correlated with Clinical Features and Prognosis in Bladder Cancer Patients}

The clinical significance of Msi2 overexpression in bladder cancer was examined by IHC in paraffin-embedded, archived clinical tumor tissue specimens obtained from 167 cases of bladder cancer. We found that Msi2 expression was markedly upregulated in bladder cancer tissues and increased with advanced clinical stage (Figure 2a). Statistical analysis revealed that the expression of Msi2 was significantly associated with tumor $(\mathrm{T})$ classification $(P=0.012)$ and node $(\mathrm{N})$ classification $(P=0.001)$. However, it was not associated with age, gender, or tumor differentiation (Table 1). Kaplan-Meier survival analysis showed that the overall survival $(P=0.008)$ and recurrence-free survival $(P=0.026)$ of patients with higher expression of Msi2 were significantly poorer than patients with lower expression of Msi2 (Figure 2b). Multivariate analysis indicated that Msi2 expression was an independent prognostic factor of patient's overall survival $(P=0.031$; Table 2).

\section{Upregulation of Msi2 Promotes Bladder Cancer Cell Migration and Invasion}

In light of the correlation between Msi2 expression and lymph node metastasis, we further investigated the effect of Msi2 on migration and invasion in established stable Msi2overexpressing J82 and EJ cells (Figure 3a). Strikingly, in wound healing assays, J82-Msi2 and TJ-Msi2 cells exhibited a significantly increased migratory ability compared with control group (Figure 3b). Similar results were found in the Boyden chamber assays, another method used to test the migration and invasion properties of tumor cells (Figure 3c). Collectively, upregulation of Msi2 promoted the migration and invasion of bladder cancer cells. 
a

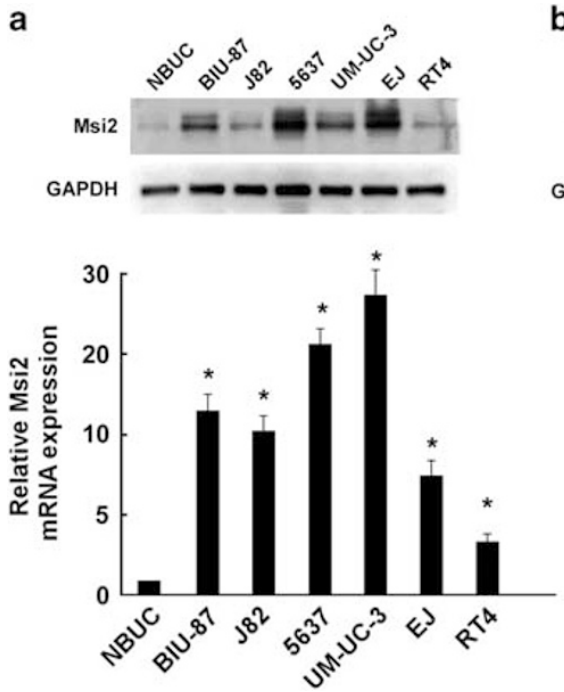

b
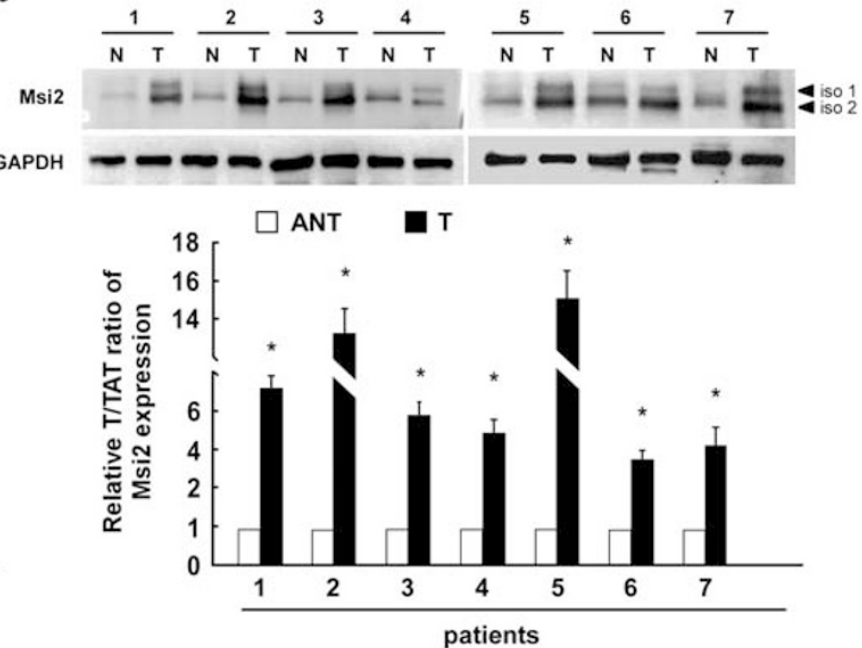

Figure 1 Msi2 expression is increased in bladder cancer cell lines and tissues. (a) Western blot and real-time PCR assay of Msi2 expression in normal bladder urothelial cells (NBUCs) and bladder cancer cell lines (BIU-87, J82, 5637, UM-UC-3, EJ, and RT4). (b) The expression of Msi2 in seven paired bladder tumor tissues (T) and paired adjacent non-tumor tissues (ANT). Msi2 expression was normalized by U6 expression. Bars represent the means of three independent experiments. ${ }^{*} P<0.05$.

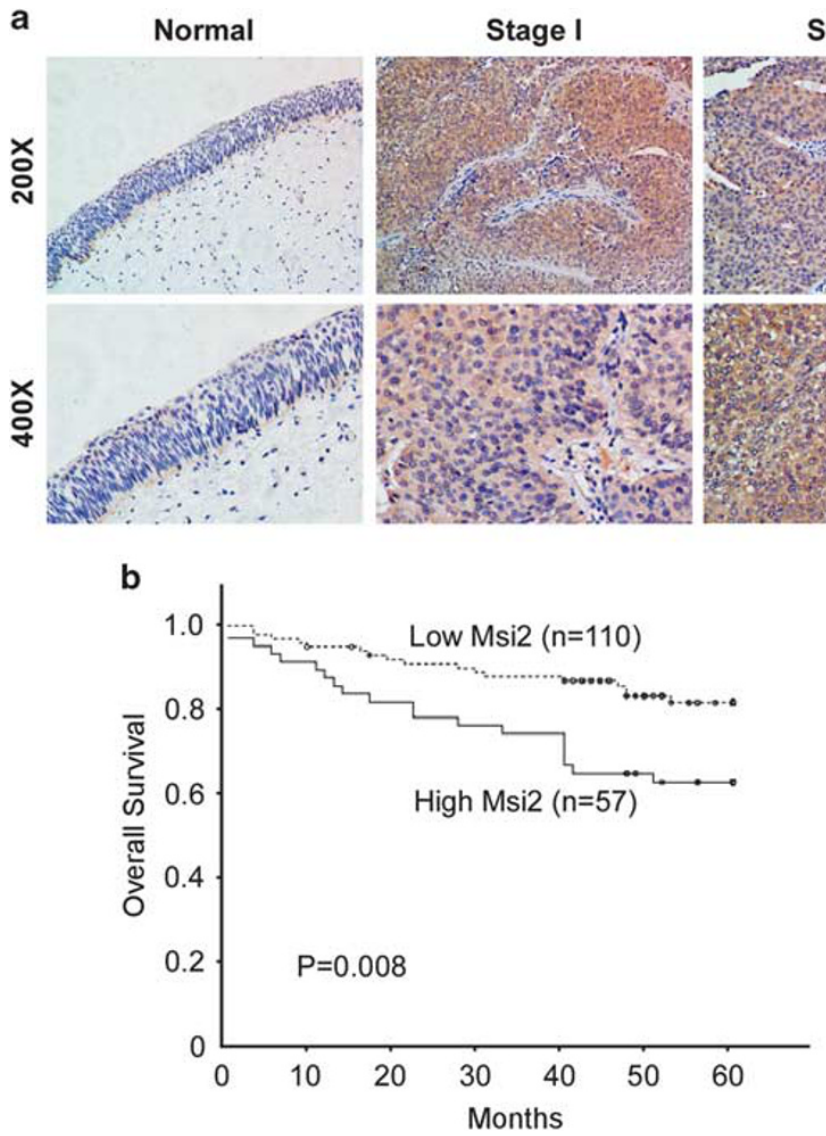

Stage II
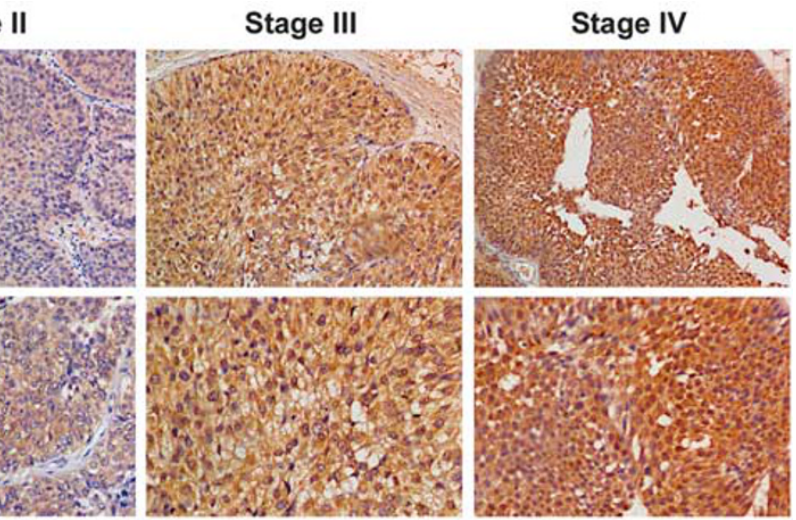

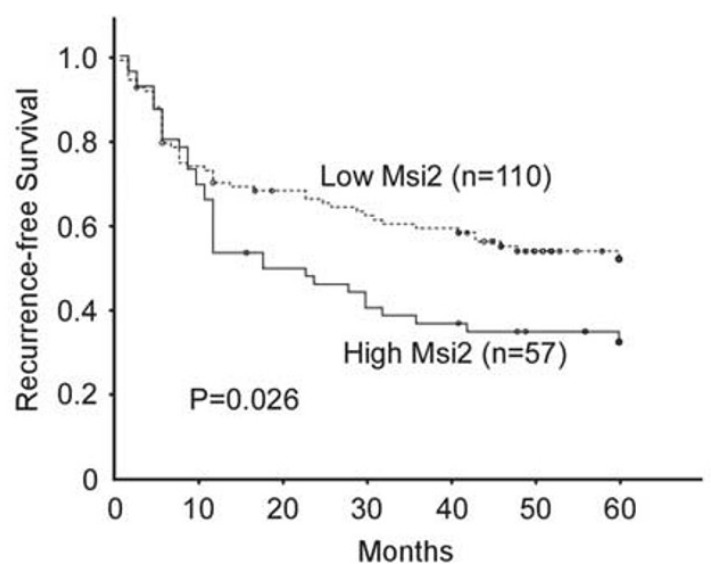

Figure 2 Msi2 expression is correlated with tumor stage and clinical prognosis in bladder cancer patients. (a) Immunohistochemical staining of Msi2 expression in human bladder (TNM stages I-IV) and normal bladder tissues. Msi2 expression was undetectable in normal bladder tissues, and was increased from stage I to stage IV. (b) Kaplan-Meier survival curves comparing cumulative overall and recurrence-free survival rates in patients with low and high Msi2 expression levels. 


\section{Msi2 Knockdown Inhibits Bladder Cancer Cell Migration and Invasion}

To further demonstrate the role of Msi2 on bladder cancer cell migration and invasion, we infected J82 and EJ cells with empty vector alone and the two shRNAs that suppressed endogenous Msi2 expression stably. Western blot analysis determined that MSI2 isoform 1 and isoform 2 were both substantially reduced after Msi2 shRNA treatment (Figure 4a). As expected, downregulation of Msi2 reduced the migratory ability of both bladder cancer cell lines as compared with the control group (Figure 4b). Moreover, the Boyden chamber

Table 1 Correlation between Msi2 expression and clinicopathological characteristics

\begin{tabular}{|c|c|c|c|c|}
\hline \multirow[t]{2}{*}{ Characteristic } & \multirow[t]{2}{*}{ No. } & \multicolumn{2}{|c|}{ Msi2 expression } & \multirow[t]{2}{*}{$P$-value } \\
\hline & & Low & High & \\
\hline Sex & & & & 0.781 \\
\hline Male & 106 & 69 (65.1\%) & 37 (34.9\%) & \\
\hline Female & 61 & $41(67.2 \%)$ & $20(32.8 \%)$ & \\
\hline Age (y) & & & & 0.525 \\
\hline$\leq 60$ & 76 & $52(68.4 \%)$ & $24(32.6 \%)$ & \\
\hline$>60$ & 91 & $58(63.7 \%)$ & $33(36.3 \%)$ & \\
\hline Differentiation & & & & 0.455 \\
\hline G1 & 77 & $53(68.8 \%)$ & $24(31.2 \%)$ & \\
\hline G2-G3 & 90 & $57(63.3 \%)$ & $33(36.7 \%)$ & \\
\hline T classification & & & & 0.012 \\
\hline $\mathrm{Ta}, \mathrm{T} 1$ & 112 & $81(72.3 \%)$ & $31(27.9 \%)$ & \\
\hline $\mathrm{T} 2-\mathrm{T} 4$ & 55 & $29(52.7 \%)$ & $26(47.3 \%)$ & \\
\hline N classification & & & & 0.001 \\
\hline$N-$ & 152 & $106(69.7 \%)$ & $46(30.3 \%)$ & \\
\hline $\mathrm{N}+$ & 15 & $4(26.7 \%)$ & $11(73.3 \%)$ & \\
\hline Total no. of patients & 167 & 110 & 57 & \\
\hline
\end{tabular}

Bold values are significant at $P<0.05$. assay were dramatically inhibited by the ablation of Msi2 (Figure 4c). Furthermore, the reduction in migration and invasion was not due to reduction in cell growth, as MTT and colony formation assays showed that Msi2 does not have a role in the proliferation of bladder cancer cells (Supplementary Figure 1). Thus, downregulation of Msi2 suppressed the migration and invasion of bladder cancer cells.

\section{Msi2 Regulated the JAK2/STAT3 Signaling Pathway in Bladder Cancer Cells}

Previous studies have demonstrated that the JAK2/STAT3 signaling pathway has a central role in tumor metastasis, ${ }^{16}$ which prompted us to investigate whether Msi2 is involved in regulation of the JAK2/STAT3 signaling pathway in bladder cancer. As shown in Figures $5 \mathrm{a}-\mathrm{c}$, overexpression of Msi2 significantly increased and silencing of Msi2 reduced the phosphorylation of JAK2 and its downstream effecter STAT3 in both cell lines. Moreover, it has been reported that activation of WASF3 by JAK2/STAT3 is the critical event promoting cell migration and invasion, ${ }^{17}$ we tested whether the WASF3 protein level was influenced by Msi2 expression in bladder cancer cells. Western blotting showed that the expression of WASF3 was significantly higher in Msi2-overexpressing tumor cells and lower in Msi2-silenced tumor cells than the respective control cells (Figure 5c). Taken together, our results suggest that Msi2 mediates migration and invasion in bladder cancer cells via functional activation of the JAK2/STAT3 signaling pathway.

\section{DISCUSSION}

The key finding in this study lies in, for the first time, the biological role of Msi2 in bladder cancer metastasis and progression. Msi2 mRNA and protein were elevated in both bladder cancer cells and clinical samples. In addition, Msi2 isoform 2 appears to be the major isoform expressed in bladder cancer cell lines and tissues, which was consistent with the expression pattern of Msi2 in glioblastoma cells and embryonic stem cells. ${ }^{18,19}$ Clinical analysis also indicated that high expression of Msi2 was correlated with poor overall and

Table 2 Multivariate analysis of overall and recurrence-free survival

\begin{tabular}{|c|c|c|c|c|}
\hline \multirow[t]{2}{*}{ Prognostic variables } & \multicolumn{2}{|c|}{ Overall survival } & \multicolumn{2}{|c|}{ Recurrence-free survival } \\
\hline & Hazard ratio $(95 \% \mathrm{Cl})$ & $P$-value & Hazard ratio $(95 \% \mathrm{Cl})$ & $P$-value \\
\hline \multicolumn{5}{|l|}{ Sex (M vs F) } \\
\hline \multicolumn{5}{|l|}{ Age $(>40$ vs $\leq 40)$} \\
\hline Differentiation (G2-3 vs G1) & $2.982(1.300-6.843)$ & 0.010 & & \\
\hline \multicolumn{5}{|l|}{ T classification (T2-4 vs T1,Ta) } \\
\hline N classification (+ vs -) & & & $2.015(1.068-3.800)$ & 0.030 \\
\hline Msi2 (high vs low) & $2.042(1.067-3.908)$ & 0.031 & & \\
\hline
\end{tabular}

Bold values are significant at $P<0.05$. 
a

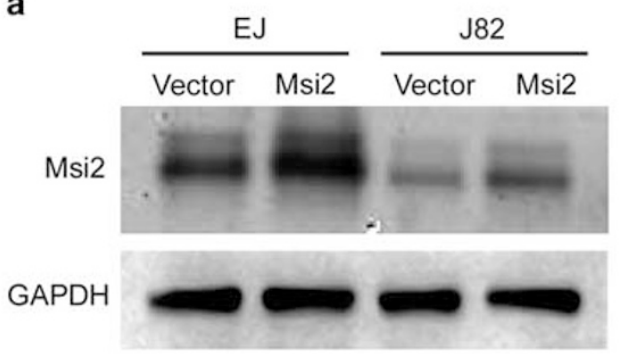

b

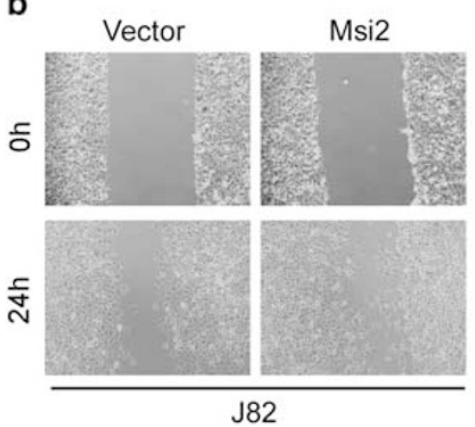

C

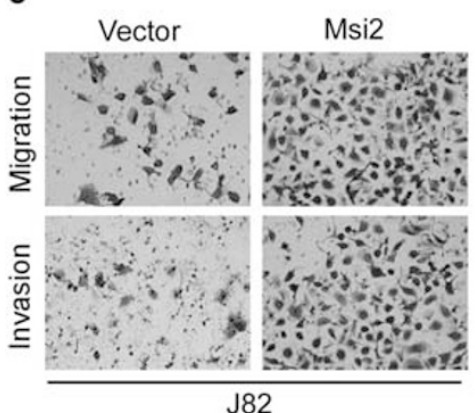

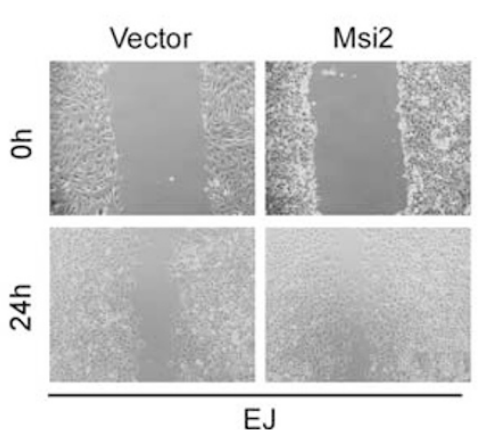

EJ

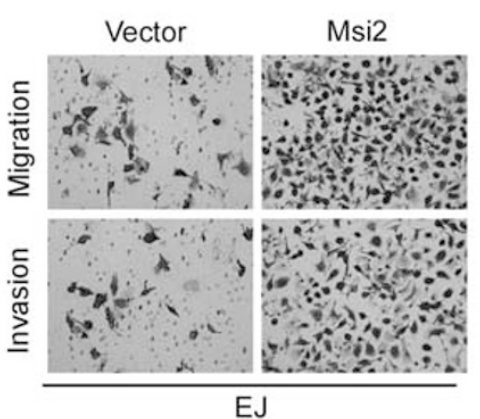

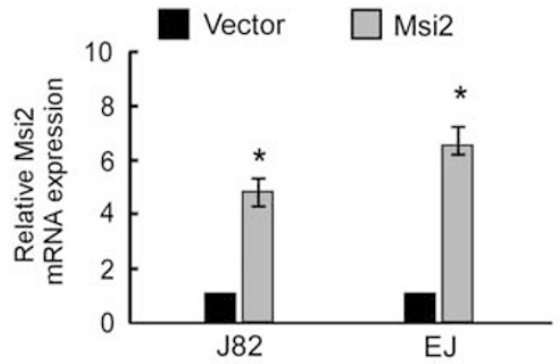
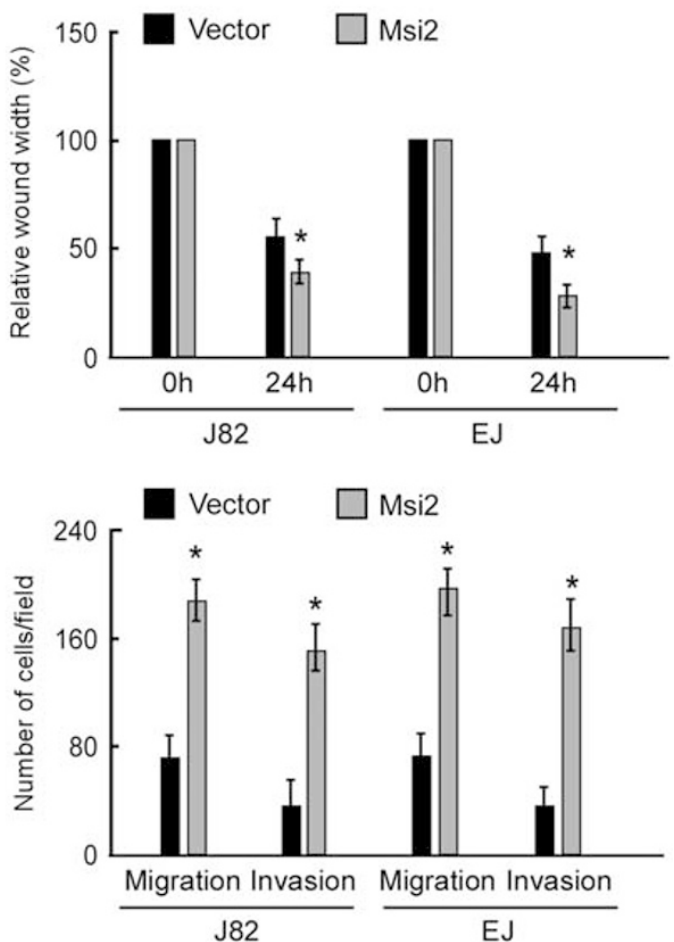

Figure 3 Msi2 overexpression promotes migration and invasion in bladder cancer cells. (a) Western blotting of Msi2 expression in vector- and Msi2transduced J82 and EJ cells; GAPDH was used as a loading control. (b) The mobility of cells was measured by wound healing assay (left panels), the uncovered areas in the wound healing assays were quantified as a percentage of the original wound area (right panels). (c) Effect of Msi2 overexpression in cell migration and invasion was determined by transwell and Matrigel assay (left panels). Quantifications of migrated cells through the membrane and invaded cells through Matrigel of each cell line are shown as proportions of their vector controls (right panels). Bar graphs show the statistical analysis of three independent experiments $\left({ }^{*} P<0.05\right)$.

recurrence-free survival in patients with bladder cancer. Moreover, in vitro assays indicated that overexpression of Msi2 promoted, while silencing of Msi2 inhibited, the migration and invasiveness of bladder cancer cell through activating JAK2/STAT3 pathway. Our results not only reveal a mechanism that promotes metastasis in bladder cancer, but also provides insight into novel therapeutic strategy in the treatment of bladder cancer patients.

Metastatic bladder cancer remains a deadly disease with limited survival time. Despite these innovations in lymphadenectomy and adjuvant chemotherapy, patients with the presence of lymph node metastasis have poorer clinical prognosis compared with those without lymphatic spread. ${ }^{20}$ Therefore, identifying molecules that detect tumor metastasis and invasion at an early stage could determine a significant improvement in morbidity and clinical outcome in bladder cancer patients. In this study, we found that overexpression of Msi2 markedly promoted cell migration and invasion in bladder cancer cells, while knocking down of Msi2 dramatically reduced bladder cancer cell migration and invasiveness. Furthermore, Msi2 expression was significantly correlated with the clinical stage and lymph nodes metastasis in bladder cancer patients, implying that Msi2 overexpression might be a central event in bladder cancer metastasis and progression. To the best of our knowledge, this is the first study revealing the relationship of Msi2 expression and tumor metastasis using both in vivo and in vitro assays.

Previous studies have shown that Msi2 expression is closely correlated with clinical prognosis of patients with hematological malignancies. Msi2 expression was negatively 
a

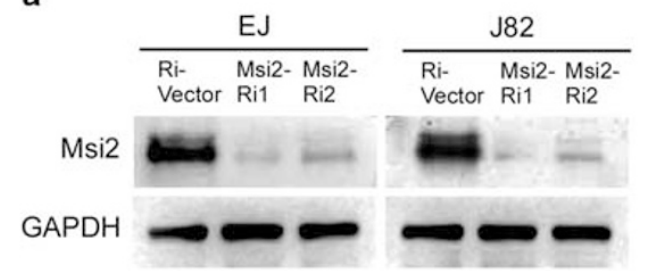

Ri-Vector $\square$ Msi2-Ri1 $\square$ Msi2-Ri2

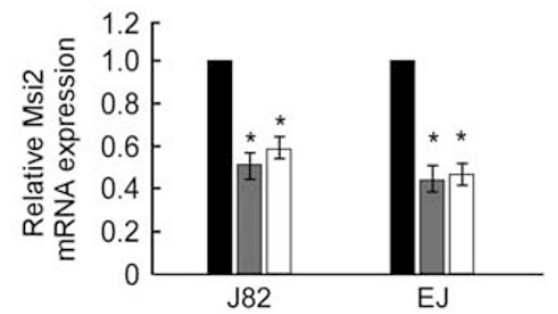

b
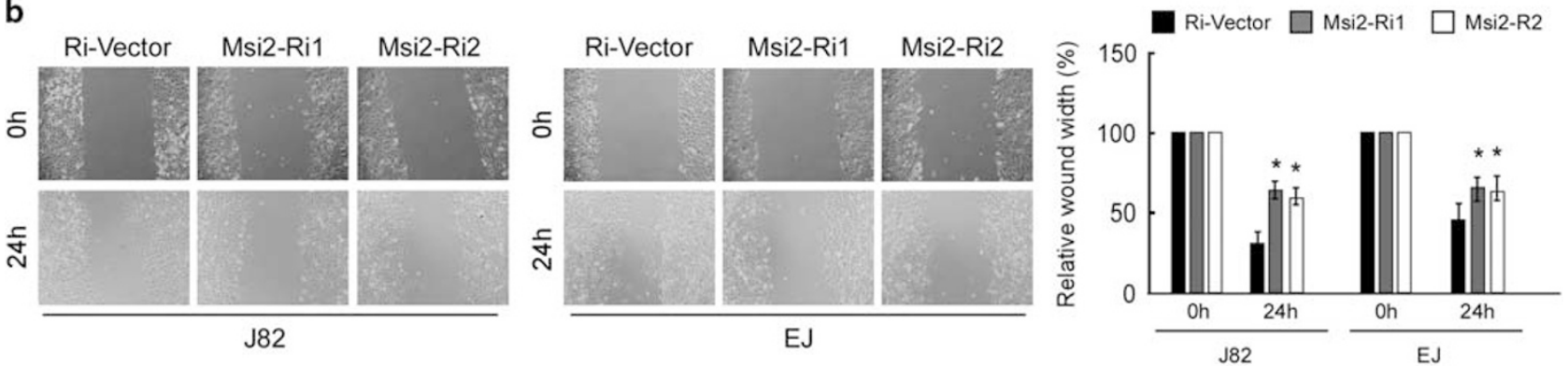

C
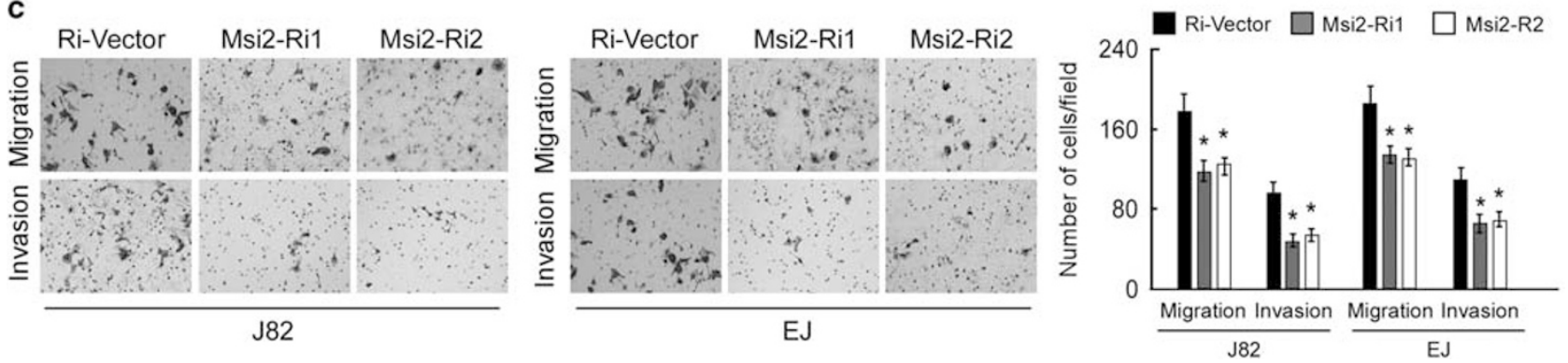

Figure 4 Msi2 silencing inhibits cell migration and invasion in bladder cancer cells. (a) Western blotting shows the levels of Msi2 protein in Msi2-silenced bladder cancer cells; GAPDH was used as a loading control. (b) The mobility of cells was measured by wound healing assay (left panels), the uncovered areas in the wound healing assays were quantified as a percentage of the original wound area (right panels). (c) Effect of Msi2 knockdown in cell migration and invasion was determined by transwell and Matrigel assay (left panels). Quantifications of migrated cells through the membrane and invaded cells through Matrigel of each cell line are shown as proportions of their vector controls (right panels). Bar graphs show the statistical analysis of three independent experiments $\left({ }^{*} P<0.05\right)$.

associated with AML patients outcome ${ }^{21}$ while the expression of NUMB, which was negatively regulated by Msi2 both in vitro and in vivo, ${ }^{19}$ was not associated with overall survival in AML patients. ${ }^{22} \mathrm{Mu}$ et al. ${ }^{23}$ reported that high Msi2 expression could indicate poor prognosis in adult B-cell acute lymphoblastic leukemia. Recently, $\mathrm{He}$ et al. unraveled the association of Msi2 expression and prognosis in patients with solid tumor. They found that Msi2 expression correlated with EMT and was a predictive biomarker of HCC prognosis. ${ }^{13}$ In agreement with their report, we have shown that Msi2 expression was upregulated in human bladder cancer tissues and cell lines, and that patients with higher levels of Msi2 expression had shorter overall and recurrent-free survival time compared with those with lower Msi2 expression. Together, these studies support the proposal that Msi2 is a novel predictor for prognosis in human malignancies.

In conclusion, Msi2, a RNA-binding protein, has been reported to inhibit the expression of some genes via binding to their mRNA, and be involved in activation of several signaling pathways. Zhang et al. ${ }^{24}$ proposed that Msi2 modulated leukemic cell proliferation and leukemogenesis involving the MAPK signaling pathway. Park et al. ${ }^{25}$ identified a novel link between Msi 2 and TGF- $\beta$ signaling pathway in regulating HSC translatome and balancing HSC homeostasis. Wang et al. ${ }^{26}$ uncovered that Msi2 promotes HCC cell invasion and progression via the $\mathrm{Wnt} / \beta$-catenin signaling pathway. Jak2/STAT3 signaling has been shown to have a pivotal role in the epithelial-mesenchymal transition (EMT) to cancer cells, ${ }^{16}$ and the importance of Jak2/STAT3 signaling in bladder cancer progression has been implicated. Zhao et al. $^{27}$ showed that activation of Jak2/STAT3 in bladder cancer cells drove EMT and tumor invasion. Joung et al. ${ }^{28}$ reported that AG490, a Jak2 inhibitor, could inhibit bladder cancer cell viability and cell migration, accompanied by reduction of STAT3 signaling. These studies indicate an oncogenic role of Msi2 in bladder cancer progression. However, the biological relationship of Msi2 and Jak2/STAT3 signaling in bladder cancer metastasis remains unclear. Here 

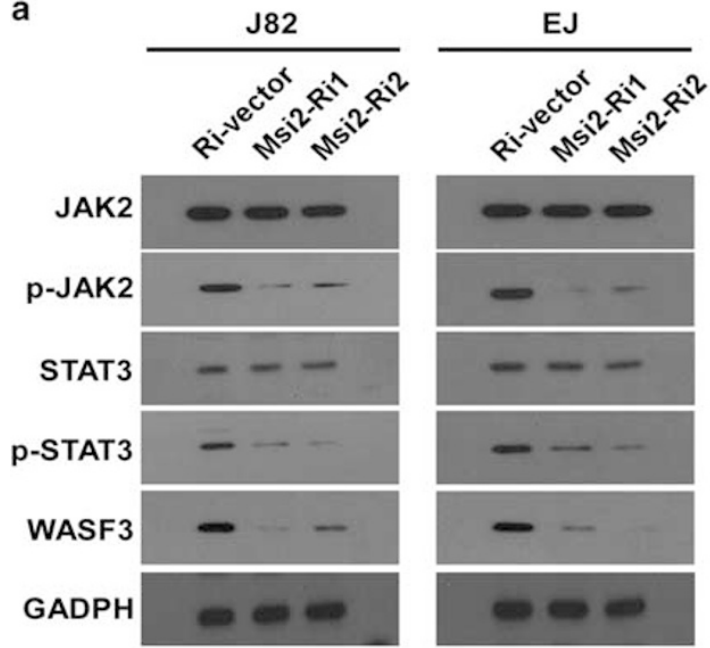

b

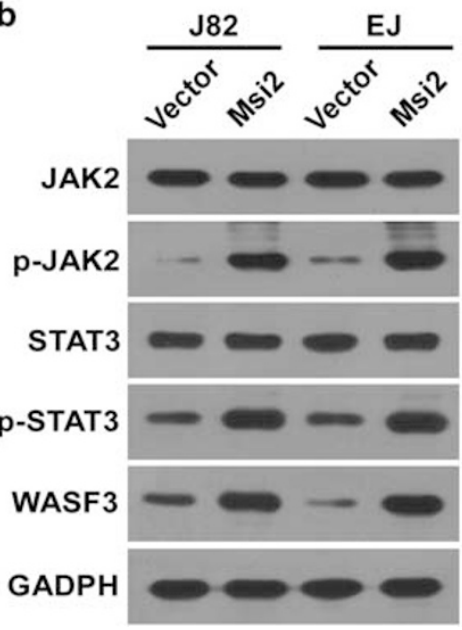

C

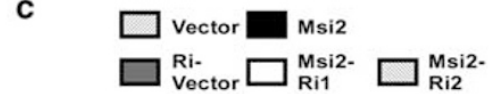

Figure 5 Msi2 activates JAK2/STAT3 signaling pathway. (a, b) Western blotting analysis indicating an apparent overlap between Msi2 expression and phosphorylation of JAK2 and STAT3, and the expression of WASF3, a key downstream effector of JAK2/STAT3 pathway. (c) Real-time PCR analysis shows WASF3 expression in Msi2-transduced and Msi2-silenced J82 and EJ cells. Bar graphs show the statistical analysis of three independent experiments $(* P<0.05)$.

we showed that overexpression of Msi2 in human bladder cells was correlated with expression of Jak2 and STAT3, and activation of genes downstream of Jak2/STAT3, indicating that Msi2 may induce metastasis and invasion in bladder cancer via Jak2/STAT3 signaling pathway. Our findings have provided new insights into the carcinogenesis of bladder cancer.

This study provides the first report demonstrating that Msi2 in patients with bladder cancer may contribute to tumor metastasis and poor prognosis, and suggested Msi2 might be a useful biomarker in early detection of bladder cancer metastasis. Moreover, we have provided a novel molecular mechanism for bladder cancer metastasis. However, further studies are needed to investigate the precise role of Msi2 in the metastatic process of bladder cancer.

\section{ACKNOWLEDGMENTS}

The study was supported by the National Natural Science Foundation of China (Grant No. 81402303).

\section{DISCLOSURE/CONFLICT OF INTEREST}

The authors declare no conflict of interest.

1. Malats N, Real FX. Epidemiology of bladder cancer. Hematol Oncol Clin North Am 2015;29:177-189 vii.

2. Kaufman DS, Shipley WU, Feldman AS. Bladder cancer. Lancet 2009;374:239-249.

3. Madersbacher S, Hochreiter W, Burkhard F, et al. Radical cystectomy for bladder cancer today-a homogeneous series without neoadjuvant therapy. J Clin Oncol 2003;21:690-696.

4. Park JC, Citrin DE, Agarwal PK, et al. Multimodal management of muscle-invasive bladder cancer. Curr Probl Cancer 2014;38:80-108.

5. Sakakibara $\mathrm{S}$, Nakamura $\mathrm{Y}$, Satoh $\mathrm{H}$, et al. Rna-binding protein Musashi2: developmentally regulated expression in neural precursor cells and subpopulations of neurons in mammalian CNS. J Neurosci 2001;21:8091-8107. 
6. De Weer A, Speleman F, Cauwelier B, et al. EVI1 overexpression in $\mathrm{t}(3 ; 17)$ positive myeloid malignancies results from juxtaposition of EVI1 to the MSI2 locus at 17q22. Haematologica 2008;93:1903-1907.

7. Siddall NA, McLaughlin EA, Marriner NL, et al. The RNA-binding protein Musashi is required intrinsically to maintain stem cell identity. Proc Natl Acad Sci USA 2006;103:8402-8407.

8. Sakakibara S, Nakamura $\mathrm{Y}$, Yoshida $\mathrm{T}$, et al. RNA-binding protein Musashi family: roles for CNS stem cells and a subpopulation of ependymal cells revealed by targeted disruption and antisense ablation. Proc Natl Acad Sci USA 2002;99:15194-15199.

9. Kharas MG, Lengner CJ, Al-Shahrour F, et al. Musashi-2 regulates normal hematopoiesis and promotes aggressive myeloid leukemia. Nat Med 2010;16:903-908.

10. Sutherland JM, Fraser BA, Sobinoff AP, et al. Developmental expression of Musashi-1 and Musashi-2 RNA-binding proteins during spermatogenesis: analysis of the deleterious effects of dysregulated expression. Biol Reprod 2014;90:92.

11. Han Y, Ye A, Zhang Y, et al. Musashi-2 silencing exerts potent activity against acute myeloid leukemia and enhances chemosensitivity to Daunorubicin. PLoS One 2015;10:e0136484.

12. Barbouti $A$, Hoglund $M$, Johansson $B$, et al. A novel gene, MSI2, encoding a putative RNA-binding protein is recurrently rearranged at disease progression of chronic myeloid leukemia and forms a fusion gene with HOXA9 as a result of the cryptic $t(7 ; 17)(p 15 ; q 23)$. Cancer Res 2003;63:1202-1206.

13. He L, Zhou X, Qu C, et al. Musashi2 predicts poor prognosis and invasion in hepatocellular carcinoma by driving epithelialmesenchymal transition. J Cell Mol Med 2014;18:49-58.

14. Hou T, Ou J, Zhao X, et al. MicroRNA-196a promotes cervical cancer proliferation through the regulation of FOXO1 and p27Kip1. $\mathrm{Br}$ Cancer 2014;110:1260-1268.

15. Hou T, Zhang W, Tong C, et al. Putative stem cell markers in cervical squamous cell carcinoma are correlated with poor clinical outcome. BMC Cancer 2015;15:785.

16. Teng $\mathrm{Y}$, Ross JL, Cowell JK. The involvement of JAK-STAT3 in cell motility, invasion, and metastasis. JAKSTAT 2014;3:e28086.
17. Teng Y, Ghoshal P, Ngoka L, et al. Critical role of the WASF3 gene in JAK2/STAT3 regulation of cancer cell motility. Carcinogenesis 2013;34: 1994-1999.

18. Wuebben EL, Mallanna SK, Cox JL, et al. Musashi2 is required for the self-renewal and pluripotency of embryonic stem cells. PLoS One 2012;7:e34827.

19. Cox JL, Wilder PJ, Gilmore JM, et al. The SOX2-interactome in brain cancer cells identifies the requirement of MSI2 and USP9X for the growth of brain tumor cells. PLoS One 2013;8:e62857.

20. Liedberg F, Mansson W. Lymph node metastasis in bladder cancer. Eur Urol 2006;49:13-21.

21. Byers RJ, Currie T, Tholouli $\mathrm{E}$, et al. MSI2 protein expression predicts unfavorable outcome in acute myeloid leukemia. Blood 2011;118: 2857-2867.

22. Thol F, Winschel C, Sonntag AK, et al. Prognostic significance of expression levels of stem cell regulators MSI2 and NUMB in acute myeloid leukemia. Ann Hematol 2013;92:315-323.

23. $\mathrm{Mu} Q$, Wang $Y$, Chen $B$, et al. High expression of Musashi-2 indicates poor prognosis in adult B-cell acute lymphoblastic leukemia. Leuk Res 2013;37:922-927.

24. Zhang H, Tan S, Wang J, et al. Musashi2 modulates K562 leukemic cell proliferation and apoptosis involving the MAPK pathway. Exp Cell Res 2014;320:119-127.

25. Park SM, Deering RP, Lu $Y$, et al. Musashi-2 controls cell fate, lineage bias, and TGF-beta signaling in HSCs. J Exp Med 2014;211 71-87.

26. Wang MH, Qin SY, Zhang SG, et al. Musashi-2 promotes hepatitis Bvirus related hepatocellular carcinoma progression via the Wnt/beta-catenin pathway. Am J Cancer Res 2015;5:1089-1100.

27. Zhao D, Besser AH, Wander SA, et al. Cytoplasmic p27 promotes epithelial-mesenchymal transition and tumor metastasis via STAT3mediated Twist1 upregulation. Oncogene 2015;34:5447-5459.

28. Joung $\mathrm{YH}, \mathrm{Na} Y \mathrm{M}$, Yoo $\mathrm{YB}$, et al. Combination of AG490, a Jak2 inhibitor, and methylsulfonylmethane synergistically suppresses bladder stumor growth via the Jak2/STAT3 pathway. Int J Oncol 2014;44:883-895. 